Check for updates

Cite this: Mater. Adv., 2021, 2, 4832
Received 2nd April 2021, Accepted 7th June 2021

DOI: $10.1039 / \mathrm{d} 1 \mathrm{ma} 00304 \mathrm{f}$

rsc.li/materials-advances

\section{Cation disorder and octahedral distortion control of internal electric field, band bending and carrier lifetime in Aurivillius perovskite solid solutions for enhanced photocatalytic activity $\dagger$}

\author{
Jaideep Malik, ${ }^{a}$ Shubham Kumar, ${ }^{a}$ Priya Srivastava, (D) ${ }^{\mathrm{b}}$ Monojit Bag ${ }^{\mathrm{bc}}$ and \\ Tapas Kumar Mandal (iD *ac
}

\begin{abstract}
The overall photocatalytic activity shown by a semiconductor photocatalyst stems from a complex interplay of several critical factors that includes light absorption, carrier recombination dynamics, charge transfer resistance, lifetime of photogenerated charge carriers and surface adsorption. While most of the factors are intrinsic to a semiconductor controlled by its structure and composition, the overall activity is determined by the dominance of a single or multiple factors. The present work examines the competing roles of these factors in the photocatalytic activity of a closely related series of Aurivillius perovskite solid solutions, $\mathrm{Bi}_{6-x} \mathrm{Sr}_{x} \mathrm{Ti}_{3+x} \mathrm{Fe}_{2-x} \mathrm{O}_{18}(x=0.75,0.5$, and 0.25$)$. The cation disorder in the intergrowth structure and octahedral distortion that control the internal electric fields in these compounds alter multiple factors responsible for enhanced photocatalytic activity. The five-layer Aurivillius perovskites forming in the non-centrosymmetric $F 2 \mathrm{~mm}$ space group with highly distorted octahedra at the terminal position and least distorted octahedra at the central perovskite layer are controlled by a second-order Jahn-Teller effect of $\mathrm{Ti}^{4+}$. While a greater extent of dye adsorption leads to faster photocatalytic degradation in $\mathrm{Bi}_{5} \mathrm{SrTi}_{4} \mathrm{FeO}_{18}$ and other Aurivillius perovskites, $\mathrm{Bi}_{5.5} \mathrm{Sr}_{0.5} \mathrm{Ti}_{3.5} \mathrm{Fe}_{1.5} \mathrm{O}_{18}(x=0.5)$ shows inferior photocatalytic activity as compared to $\mathrm{Bi}_{5.75} \mathrm{Sr}_{0.25} \mathrm{Ti}_{3.25} \mathrm{Fe}_{1.75} \mathrm{O}_{18}(x=0.25)$ and $\mathrm{Bi}_{5.25} \mathrm{Sr}_{0.75} \mathrm{Ti}_{3.75} \mathrm{Fe}_{1.25} \mathrm{O}_{18}$ $(x=0.75)$ despite having the highest dye adsorption in the series. The highest rate of photocatalytic RhB degradation in the $\mathrm{Fe}_{1.75}$ compound is due to its longest average carrier lifetime in the series, sluggish $\mathrm{e}^{-}-\mathrm{h}^{+}$recombination and lower charge transfer resistance. Furthermore, the enhanced activity observed under external bias is understood to have originated from a greater upward band bending effect, which acts as thermal barrier for $\mathrm{e}^{-}-\mathrm{h}^{+}$recombination and facilitates effective charge separation and transfer. The work elucidates the delicate interplay of the competing factors affecting the photocatalytic activity and establishes the origin of non-uniform and enhanced photocatalytic activity across the homologous solid solution series of layered perovskites. The protocol will help breaking compositional limits in uncovering new solid-solution compounds with enhanced activity by appropriate substitutions and further enhancement under external bias for faster removal and degradation of pollutants.
\end{abstract}

\section{Introduction}

Increasing energy demands, growing population and depleting fossil fuel reserves compel us to look for renewable and clean resources of energy. With abundant sunlight reaching Earth's surface throughout the year, harvesting solar energy, a clean

\footnotetext{
${ }^{a}$ Department of Chemistry, Indian Institute of Technology Roorkee,

Roorkee 247 667, India. E-mail: tapas.mandal@cy.iitr.ac.in

${ }^{b}$ Department of Physics, Indian Institute of Technology Roorkee, Roorkee 247 667, India

${ }^{c}$ Centre of Nanotechnology, Indian Institute of Technology Roorkee, Roorkee 247 667, India

$\dagger$ Electronic supplementary information (ESI) available. See DOI: 10.1039/d1ma00304f
}

and renewable resource, can be the ultimate solution. ${ }^{1}$ One requires efficient devices to harness the abundant solar energy. Semiconductor photocatalysis have shown immense potential in energy generation and environmental remediation using solar energy. ${ }^{2,3}$ Photocatalysts with extended visible-light absorption can harvest maximum sunlight and may have wide ranging applications in the area of hydrogen production by water splitting, ${ }^{4}$ degradation or complete mineralization of organic pollutants in wastewater ${ }^{5,6}$ and hydrogen peroxide generation. ${ }^{7,8}$

Since the electrochemical decomposition of water at the $\mathrm{TiO}_{2}$ electrode by Fujishima and Honda in $1972,{ }^{9}$ research based on semiconductor photocatalysis for the development of new photocatalysts has surged. For the last several decades, 
metal-oxide based semiconductor materials have been widely used for photocatalytic degradation. ${ }^{10,11}$ Most of them are $\mathrm{TiO}_{2}$ based semiconductors due to their availability, stability and non-hazardous nature. ${ }^{12,13}$ However, they cannot be used under visible-light due to their large band gaps falling mainly in the UV region making them "inefficient" as the UV region only accounts for $3-5 \%$ of the incoming solar energy. Thus, novel visible-light-driven photocatalysts absorbing in the visible region with a wider absorption range to utilize maximum amounts of sunlight is indispensable. Many visible-light-driven photocatalysts were studied in recent years with layered compounds getting huge attraction. ${ }^{5,14-27}$ Recently, photocatalyst designs with self-healing internal electric fields have been demonstrated exhibiting accelerated photocatalysis. ${ }^{28}$ Moreover, a gradient doping of $\mathrm{P}$ in $\mathrm{CdS}$ was realized to create a built-in internal electric field to facilitate charge carrier extraction and enhance $\mathrm{H}_{2}$ evolution. ${ }^{29}$ Similarly, a heterojunction design with a graded solid-solution interface in $\mathrm{Mn}_{x} \mathrm{Cd}_{1-x} \mathrm{~S}$ facilitated efficient separation and transfer of charge carriers due to a gradient built-in electric field resulting excellent photocatalytic hydrogen generation. ${ }^{30}$

Compounds with the perovskite structure, especially the layered variants have shown tremendous research thrust in visible-light-driven photocatalysis..$^{5,19,31,32}$ The layered compounds, largely Aurivillius phases, attract a lot of attention for their improved separation of charge carriers due to the presence of internal electric fields ${ }^{33}$ resulting in enhanced photocatalytic activity. The Aurivillius phases are a part of a large family of layered perovskites with regular fluorite-like $\left(\mathrm{Bi}_{2} \mathrm{O}_{2}\right)^{2+}$ layers sandwiched between the perovskite $\left(\mathrm{A}_{n-1} \mathrm{~B}_{n} \mathrm{O}_{3 n+1}\right)^{2-}$ blocks, where $n$ represents the number of corner connected octahedral layers. ${ }^{34,35}$ There are many reports of Aurivillius phases related to the study of their ferroelectric and dielectric properties ${ }^{36-40}$ but only some of them were studied for their photocatalytic properties..$^{20,32,41-44}$ Out of these, a very limited number of semiconductor photocatalysts are active in the visible region of the solar spectrum. The layered compounds are modified by substitution at the A-site as well as the B-sites of the perovskite block to extend their absorption to the visible region. The compositional substitution in layered Aurivillius phases were studied most intensely for $n=1 \mathrm{mem}$ bers of the family, namely, $\mathrm{Bi}_{2} \mathrm{WO}_{6}$ and $\mathrm{Bi}_{2} \mathrm{MoO}_{6} \cdot{ }^{45-51}$ On the contrary, higher order members (with $n=2,4$ and 5) were limited and few were studied for their photocatalytic activities. $5,19,31,52-54$ Aurivillius phases, which always contain $\mathrm{Bi}$, are noteworthy because the presence of $\mathrm{Bi}$ pushes the top of the valence band upward and often shifts the absorption edge in the higher wavelength or visible region when compared with their nonbismuth counterparts, thus enhancing the catalytic activities of the compounds under visible-light irradiation. Recently, fivelayer $\mathrm{Bi}_{6} \mathrm{Ti}_{3} \mathrm{Fe}_{2} \mathrm{O}_{18}$ intrigued researchers towards its optical and ferroelectric properties, and the rare earth ( $\mathrm{La}, \mathrm{Sm}, \mathrm{Gd}$, and Dy) substituted $\mathrm{Bi}_{6} \mathrm{Ti}_{3} \mathrm{Fe}_{2} \mathrm{O}_{18}$ for their magnetic and magnetoelectric properties. $^{55,56}$

Considering that the five-layer $\mathrm{Bi}_{6} \mathrm{Ti}_{3} \mathrm{Fe}_{2} \mathrm{O}_{18}$ crystallizes in a non-centrosymmetric space group and substitution at the A-site as well as the B-sites of the perovskite would alter the strength of internal electric fields, we envisaged various compositions $\mathrm{Bi}_{6-x} \mathrm{Sr}_{x} \mathrm{Ti}_{3+x} \mathrm{Fe}_{2-x} \mathrm{O}_{18}(x=0.75,0.5$, and 0.25$)$ that fall between the two end members $\mathrm{Bi}_{6} \mathrm{Ti}_{3} \mathrm{Fe}_{2} \mathrm{O}_{18}$ and $\mathrm{Bi}_{5} \mathrm{SrTi}_{4} \mathrm{FeO}_{18}$. The compositions are attained by altering the amount of $\mathrm{Fe}$ with respect to $\mathrm{Ti}$ and counterbalancing the charge by replacing $\mathrm{Bi}$ with Sr. It is believed that the compositional manipulation would change the extent of octahedral distortion, the strength of internal electric field and charge carrier lifetime in addition to subtle changes in the band structure of the compounds, and thus help understanding the role of various factors affecting photocatalytic activity in the closely related solid-solution series. The interplay of various factors responsible for photocatalytic activity and their relative dominance or suppression in affecting the photocatalytic activity of $\mathrm{Bi}_{6-x} \mathrm{Sr}_{x} \mathrm{Ti}_{3+x} \mathrm{Fe}_{2-x} \mathrm{O}_{18}(x=0.75,0.5$, and 0.25 ) are reported. The study highlights the effect of photocatalytic activity in the presence of bias voltage and its consequences under simulated solar radiation.

\section{Experimental}

\section{Materials and synthesis}

For the synthesis, $\mathrm{Bi}_{2} \mathrm{O}_{3}$ ( $\geq 98 \%$ ), $\mathrm{SrCO}_{3}$ ( $\geq 99.9 \%$ ), $\mathrm{TiO}_{2}(99.8 \%)$ and $\mathrm{FeC}_{2} \mathrm{O}_{4} \cdot 2 \mathrm{H}_{2} \mathrm{O}(99 \%)$ were obtained from Sigma-Aldrich and used directly as supplied. All analytical grade reagents were used where required without any further purification in all our experiments and Millipore (Bedford, MA, USA) water used for preparing all the dye solutions.

The five-layered Aurivillius phases, $\mathrm{Bi}_{6-x} \mathrm{Sr}_{x} \mathrm{Ti}_{3+x} \mathrm{Fe}_{2-x} \mathrm{O}_{18}$ $(x=1.0,0.75,0.5,0.25$, and 0.0$)$, were prepared by conventional solid-state reactions. For this, stoichiometric amounts of $\mathrm{Bi}_{2} \mathrm{O}_{3}$, $\mathrm{SrCO}_{3}, \mathrm{TiO}_{2}$ and $\mathrm{FeC}_{2} \mathrm{O}_{4} \cdot 2 \mathrm{H}_{2} \mathrm{O}$ were ground in an agate mortar for $1 \mathrm{~h}$ and the powder mixture was fired at $780{ }^{\circ} \mathrm{C}$ for $2 \mathrm{~h}$. Then, the mixture was ground again and pressed into pellets of $12 \mathrm{~mm}$ diameter. These pellets were reheated at $1000{ }^{\circ} \mathrm{C}$ for different times with intermediate grinding as given in Table S1 (ESI $\dagger$ ).

\section{Characterization}

Powder X-ray diffraction (PXRD) analysis. The progress of the reaction and phase formation after each step was monitored by PXRD analysis. The PXRD measurements were carried out on a Bruker AXS D8 Advance diffractometer operated at $40 \mathrm{kV}$ and 30 mA using graphite monochromatized $\operatorname{CuK}_{\alpha}(\lambda=1.5406 \AA)$ radiation in the angular range of $5-90^{\circ}$. The phase purity of synthesized compounds was determined by matching the observed diffraction patterns with the standard PDFs available in the JCPDS (Joint Committee on Powder Diffraction Standards) database and literature. PROSZKI program ${ }^{57}$ was used for least-squares refinement of lattice parameters of $\mathrm{Bi}_{6-x} \mathrm{Sr}_{x} \mathrm{Ti}_{3+x} \mathrm{Fe}_{2-x} \mathrm{O}_{18} \quad(x=1.0$, $0.75,0.5,0.25$, and 0.0$)$. Rietveld refinements of PXRD data were carried out using the Fullprof program suite. ${ }^{58}$ For these refinements, the PXRD were recorded in the range $5^{\circ} \leq 2 \theta \leq 90^{\circ}$ with a step size of $0.018^{\circ}$ for $5 \mathrm{~h}$.

In the earlier stages of structure refinement, scale factor and cell parameters were refined followed by background coefficients along with zero shift. In the successive runs shape 
and other profile parameters were refined systematically to avert any divergence. Positional parameters were refined just before the thermal parameters in the later stages of the refinement based on the atomic weights of elements, i.e., by choosing the heavier one first while positional parameters of oxygen were refined last as it is the lightest atom, although its thermal parameters were left unchanged to avoid unrecoverable divergence. Finally, refinement by varying the occupancy of atoms was performed to examine cation disorder by fixing refined positions and thermal parameters of the atoms.

Field emission-scanning electron microscopy (FE-SEM) and energy dispersive spectroscopy (EDS) analysis. Morphology of the powder samples were studied using a Carl Zeiss FE-SEM, Ultra plus55, operated at an accelerating voltage of $20 \mathrm{kV}$. The elemental compositions and mapping analysis of the samples were done using an energy dispersive X-ray spectroscope (EDS) (Oxford Instruments) attached to the FE-SEM instrument. For this, the sample was prepared by smearing the powdered compounds on a carbon tape pasted on a metal sub and then a thin layer of $\mathrm{Au}$ was sputtered on it for electrical conductivity. Spot and area analysis were performed to ascertain the elemental compositions in different regions of the sample to determine local as well as overall compositional homogeneity.

High-resolution-transmission electron microscopy (HR-TEM) analysis. High-resolution transmission electron microscopy (HR-TEM) images and selected area electron diffraction (SAED) patterns were recorded using a JEM-3200FS field-emission electron microscope working at $300 \mathrm{kV}$. A very small amount of the sample $(\sim 0.1 \mathrm{mg})$ was dispersed in ethanol by grinding in a pestle and mortar for $10 \mathrm{~min}$. Then, a small amount of the suspension was taken in a micropipette and drop cast on a carbon-coated copper grid and allowed to dry in air.

Ultraviolet-visible diffuse reflectance spectroscopy (UV-vis DRS) analysis. The optical properties of the compounds were measured by UV-visible diffuse reflectance spectroscopy (DRS). It involves measurement of the relative change in diffuse reflected light compared with that of $\mathrm{BaSO}_{4}$ used as reference material in all possible directions from particles oriented in all directions. For this, a Shimadzu UV-2450 UV-vis spectrophotometer was used and the UV-vis DRS data were recorded in the wavelength range $200-800 \mathrm{~nm}$. The reflectance data were converted into absorption and band gaps were calculated using the standard equations and methods given in the ESI. $\dagger$

Photoluminescence (PL) analysis. Upon photoexcitation of electrons in a semiconductor, the electrons get excited from the valence band to the conduction band and when they spontaneously de-excite they emit light; this combined process is called photoluminescence. Room temperature PL measurements were carried out on a Horiba Fluoromax- 4 spectrofluorophotometer in the wavelength range $400-550 \mathrm{~nm}$ at an excitation wavelength of $355 \mathrm{~nm}$. For PL studies, first, the powder sample was filled in the groove of a sample holder; it was placed in the spectrofluorophotometer, and the PL spectra were recorded. Fluorescence lifetime spectroscopy (FLS) was utilized to measure the average lifetime of photogenerated charge species. For this, suspensions of samples in solutions of $\mathrm{pH} 2.4$ were prepared and decay spectra was recorded on a Horiba Jobin Yvon FluoroCube fluorescence spectrophotometer at an emission wavelength of $470 \mathrm{~nm}$.

$\zeta$-Potential. $\zeta$-Potential is the measure of the potential difference existing between the surface of a solid particle immersed in a conducting liquid and the bulk of the liquid. $\zeta$-Potential measurements were carried out using a Malvern Zetasizer Nano ZS90. Samples were prepared by suspending $10 \mathrm{mg}$ of solid sample in $10 \mathrm{~mL}$ aqueous solution of different $\mathrm{pH}(2,3,5,7,9$ and 11).

\section{Photocatalytic activity study}

Adsorption studies. For adsorption study, $100 \mathrm{mg}$ of the catalyst (as the adsorbent) was dispersed in $100 \mathrm{~mL}$ of $1 \times$ $10^{-5} \mathrm{M}$ RhB dye solution of a relevant $\mathrm{pH}(2,7$ and 11$)$. Then this dye-photocatalyst suspension was magnetically stirred at a rate of $420 \mathrm{rpm}$ for $6 \mathrm{~h}$ in the dark and the absorbance was measured using a Shimadzu 2450 UV-visible spectrophotometer by taking aliquots of the suspension at regular intervals. The percentage of dye adsorbed was calculated using the equation given below:

$$
\text { Adsorption }(\%)=\left(1-C / C_{0}\right) \times 100
$$

where $C$ is the concentration of the dye at any time interval and $C_{0}$ is the initial dye concentration.

Photocatalysis. Photocatalytic activity was investigated via degradation of the dye (RhB) under solar irradiation at different $\mathrm{pH}$. These photocatalysis experiments were performed at IIT Roorkee during September-October with Direct Normal Irradiance $\sim 220-188 \mathrm{~W} \mathrm{~m}^{-2}$. For this, $0.1 \mathrm{~g}$ of photocatalyst was added into $100 \mathrm{~mL}$ of $1 \times 10^{-5} \mathrm{M}$ RhB dye solution. Then, the dyephotocatalyst suspension was magnetically stirred in the dark for $3 \mathrm{~h}$ to attain adsorption-desorption equilibrium (optimized by adsorption test studies) and subsequently irradiated with sunlight. Change in the dye concentration during degradation was measured by taking an aliquot $(\sim 4 \mathrm{~mL})$ of dye suspension at regular time intervals, centrifuging it at $10000 \mathrm{rpm}$ to separate suspended catalyst and recording the absorbance of the centrifugate on a Shimadzu 2450 UV-visible spectrophotometer. A blank experiment was also performed under similar conditions to account for any correction due to self-degradation of the dye.

Catalyst dosage test. A test was performed to optimize the catalyst amount required to achieve maximum efficiency dosage. For this experiment, varying amounts of catalysts $(2.5,5,7.5,10$, $12.5,15$ and $17.5 \mathrm{mg}$ ) were dispersed in $10 \mathrm{~mL}$ of $1 \times 10^{-5} \mathrm{M} \mathrm{RhB}$ dye solution. Then these dye-photocatalyst suspensions were sonicated for 5 minutes and irradiated under sunlight for 10 minutes. After irradiation with light, these suspensions were centrifuged to separate the catalyst and absorbance of these solutions was measured on an UV-vis spectrophotometer (Fig. S1 in the ESI $\dagger$ ).

Analysis of reactive species. Reactive species playing leading roles in the degradation of the $\mathrm{RhB}$ dye over the $\mathrm{Bi}_{5.75} \mathrm{Sr}_{0.25^{-}}$ $\mathrm{Ti}_{3.25} \mathrm{Fe}_{1.75} \mathrm{O}_{18}$ catalyst under sunlight were determined using scavengers that were added into the suspension. Ammonium oxalate (AO) for holes $\left(\mathrm{h}^{+}\right)$, benzoquinone (BQ) for superoxide 
radical anions $\left(\mathrm{O}_{2}{ }^{--}\right)$and tertiary butyl alcohol $(t-\mathrm{BuOH})$ for hydroxyl radicals $\left({ }^{\bullet} \mathrm{OH}\right)$ were used as scavengers in our experiments. All the experiments were performed in similar way to that illustrated in the photocatalysis studies.

\section{Electrochemical measurements}

Electrochemical impedance spectroscopy (EIS) studies were carried out on a Multi Autolab/M204 electrochemical workstation (Metrohm Autolab B.V., Netherlands) with a threeelectrode assembly. For this, Pt wire was used as the counter electrode, $\mathrm{Ag} / \mathrm{AgCl}$ was used as the reference electrode and the photocatalyst material coated on ITO was used as the working electrode in a $10 \mathrm{mM} \mathrm{K}_{3}\left[\mathrm{Fe}(\mathrm{CN})_{6}\right]$ solution containing $0.1 \mathrm{M} \mathrm{KCl}$ as the electrolyte while the EIS study under illumination at biased voltage was carried out on a Zahner Electrochemical Workstation in the frequency range of $100 \mathrm{mHz}$ to $1 \mathrm{MHz}$ under the light intensity of $400 \mathrm{~W} \mathrm{~m}^{-2}$ at 0 and $1 \mathrm{~V}$ in a two-electrode system. For this, $\mathrm{Pt}$ wire was used as the counter electrode, the photocatalyst coated ITO was used as the working electrode and tetrabutylammonium hexafluorophosphate (TBAPF6) in dichloromethane (DCM) was used as the liquid electrolyte. Working electrodes were prepared by mixing compounds with $2 \%$ of PVDF (polyvinylidene difluoride) in NMP ( $N$-methyl-2-pyrrolidone) to make a slurry, which was then coated on ITO and dried overnight at $60{ }^{\circ} \mathrm{C}$. Experimental data were fitted with equivalent electrical circuits using ZView ${ }^{\circledR}$ software from Scribner Associates to estimate various parameters.

\section{Results and discussion}

\section{PXRD analysis}

The observed PXRD patterns (Fig. 1) of $\mathrm{Bi}_{6-x} \mathrm{Sr}_{x} \mathrm{Ti}_{3+x} \mathrm{Fe}_{2-x} \mathrm{O}_{18}$ $(x=1.0,0.75,0.5,0.25$, and 0.0$)$ indicate the formation of a fivelayer Aurivillius phase which is in agreement with the JCPDS PDF \#21-0101 and earlier data reported in the literature for fivelayer phases. ${ }^{19,59}$ All the reflections present in a pattern are indexable in the $F 2 \mathrm{~mm}$ (no. 42) space group. The least squares refined unit cell parameters (Table $\mathrm{S} 2$ in ESI $\dagger$ ) show an increase

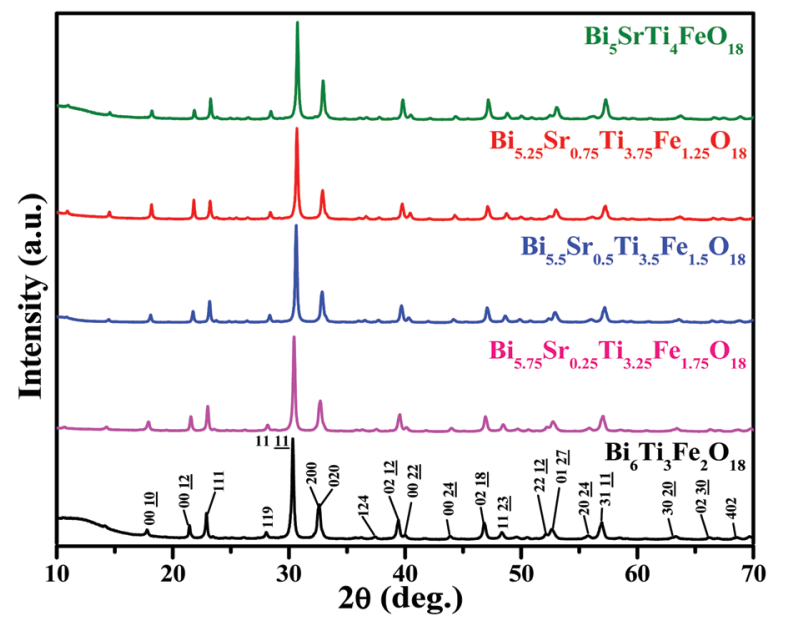

Fig. 1 PXRD patterns of $\mathrm{Bi}_{6-x} \mathrm{Sr}_{x} \mathrm{Ti}_{3+x} \mathrm{Fe}_{2-x} \mathrm{O}_{18}(x=1.0,0.75,0.5,0.25,0.0)$. in the $c$-parameter for the compounds in the series with increasing Fe from 1.25 to 1.75.

Rietveld structure refinements of the compounds are carried out using the FullProf program suite using the model structure of $\mathrm{Bi}_{6} \mathrm{Ti}_{3} \mathrm{Fe}_{2} \mathrm{O}_{18}$, where one complete layer of $\mathrm{Fe}$ is confined to the central perovskite layer and the remaining Fe (0.25, 0.5 and 0.75) are statistically distributed over the terminal $\operatorname{Ti}(1)$ and pre-terminal Ti(2)-sites. During the trial runs with all Fe distributed over all the octahedral sites and Sr over the A-sites of perovskite as well as the $\left[\mathrm{Bi}_{2} \mathrm{O}_{2}\right]^{2+}$ layers, the refinement resulted in higher values of the reliability factors. The observed, calculated and difference profiles with the lowest $\chi^{2}$ value are shown in Fig. 2. The refined atomic positions, occupancies and thermal parameters are given in the Tables S3-S5 (ESI $\dagger$ ) and the crystal structure drawn from the refined parameters for a representative member, $\mathrm{Bi}_{5.75} \mathrm{Sr}_{0.25} \mathrm{Ti}_{3.25} \mathrm{Fe}_{1.75} \mathrm{O}_{18}$, is shown in Fig. 2 . The Aurivillius perovskites form in the non-centrosymmetric F2mm space group with the occupation of one $\mathrm{Fe}$ in the central octahedral layer while the remaining Fe's are statistically distributed over the pre-terminal and terminal octahedral layers (ignored in the structural presentation). The distortions of octahedra are visible for regular $\mathrm{FeO}_{6}$ octahedra at the central layer and distorted $\mathrm{Ti} / \mathrm{FeO}_{6}$ octahedra at the pre-terminal and terminal positions with out-of-centre displacement of the $\mathrm{Ti} / \mathrm{Fe}$.

The B-cation displacement in the pre-terminal and terminal octahedra give rise to the short and long bond sequence along the $c$-axis direction (see later for detail). This also leads to the buckling of in-plane bonds at the pre-terminal and terminal octahedra. Because of this, the centre of symmetry of $\mathrm{BO}_{6}$
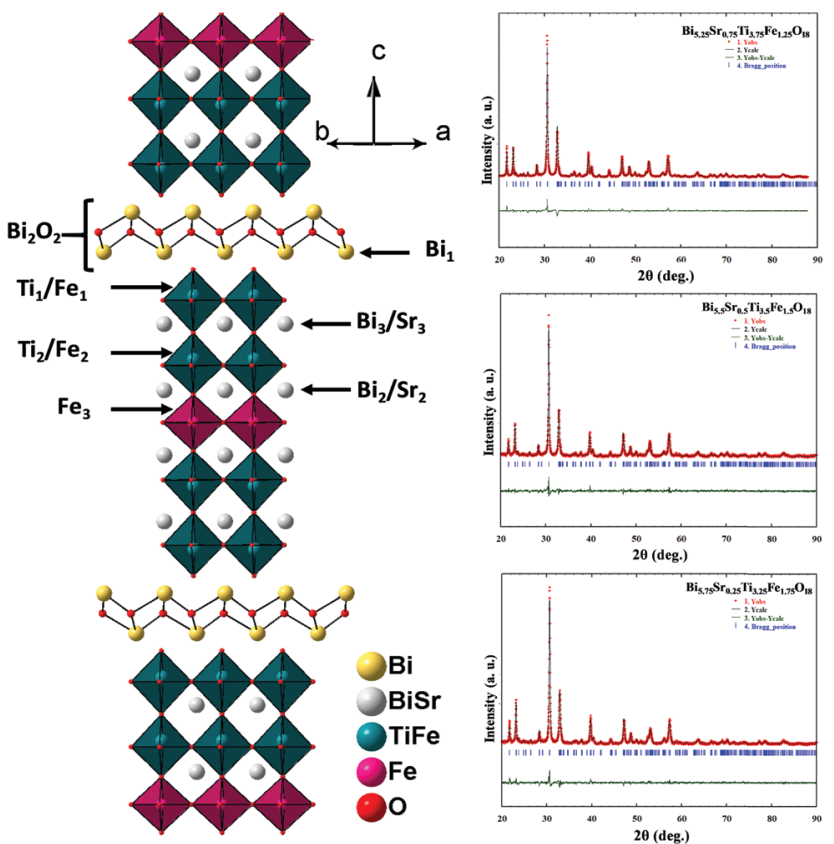

Fig. 2 Rietveld refinement profiles of $\mathrm{Bi}_{5.25} \mathrm{Sr}_{0.75} \mathrm{Ti}_{3.75} \mathrm{Fe}_{1.25} \mathrm{O}_{18}, \mathrm{Bi}_{5.5} \mathrm{Sr}_{0.5^{-}}$ $\mathrm{Ti}_{3.5} \mathrm{Fe}_{1.5} \mathrm{O}_{18}$ and $\mathrm{Bi}_{5.75} \mathrm{Sr}_{0.25} \mathrm{Ti}_{3.25} \mathrm{Fe}_{1.75} \mathrm{O}_{18}$ (right panel). Observed (+), calculated (-) and difference (bottom) profiles are shown, Vertical bars represent Bragg positions. The crystal structure of $\mathrm{Bi}_{5.75} \mathrm{Sr}_{0.25} \mathrm{Ti}_{3.25} \mathrm{Fe}_{1.75} \mathrm{O}_{18}$ constructed from the refined parameters is shown in the left panel. 
octahedra is lost, leading to the development of local non-zero polarization at the octahedra while in a centrosymmetric structure, the dipoles cancel each other resulting in the loss of overall polarization. In compounds with non-centrosymmetric space groups the resultant dipoles persist causing permanent polarization in the compounds.

\section{FE-SEM, EDS and elemental mapping analysis}

The FE-SEM images of $\mathrm{Bi}_{6-x} \mathrm{Sr}_{x} \mathrm{Ti}_{3+x} \mathrm{Fe}_{2-x} \mathrm{O}_{18}(x=0.75,0.5,0.25)$ (Fig. S2 in the ESI $\dagger$ ) show aggregated particles dominated by plate-like crystallites for all the compounds with the particle size ranging from hundreds of nanometers to few micrometers. The features are same throughout as seen in the images taken at several regions of the sample. The average calculated elemental ratios from EDS are in good agreement with the nominal compositions within admissible error limits. Elemental mapping is performed to look at the elemental distribution in $\mathrm{Bi}_{6-x^{-}}$ $\mathrm{Sr}_{x} \mathrm{Ti}_{3+x} \mathrm{Fe}_{2-x} \mathrm{O}_{18}(x=0.75,0.50,0.25)$ in different areas of the powder samples. The EDS elemental maps indicate a homogeneous distribution of the constituent elements in all the cases as shown in Fig. 3. An increase in the quantity of Bi and Fe and decrease in the quantity of $\mathrm{Sr}$ and Ti as $x$ varies from 0.75 to 0.25 can be clearly seen in the elemental maps.

\section{HR-TEM and EDS analysis}

The lattice periodicity and crystalline structure is further analysed with TEM. The cross-sectional HR-TEM image (Fig. 4a) reveals the intergrowth of Aurivillius structure with $\left[\mathrm{Bi}_{2} \mathrm{O}_{2}\right]^{2+}$ units interleaving between the five-layer perovskite slabs as seen clearly by the contrast of lattice fringes. A lattice distance of $4.91 \mathrm{~nm}$ between the marked fringes corresponds to the $c$-parameter periodicity of $\mathrm{Bi}_{5.75} \mathrm{Sr}_{0.25} \mathrm{Ti}_{3.25} \mathrm{Fe}_{1.75} \mathrm{O}_{18}$ and is in agreement with the calculated distance obtained from the PXRD. Moreover, the SAED pattern (Fig. 4b) shows single crystalline diffraction patterns and some of the bright spots are indexable corresponding to specific $h k l$ planes allowed by the $F 2 \mathrm{~mm}$ space group and in agreement with the PXRD data.

\section{UV-vis DRS analysis}

Fig. S3 (ESI $\dagger$ ) shows the UV-vis DRS in absorption term and the corresponding Tauc plots for the compounds. The compounds show two-step absorption feature with stronger and sharper

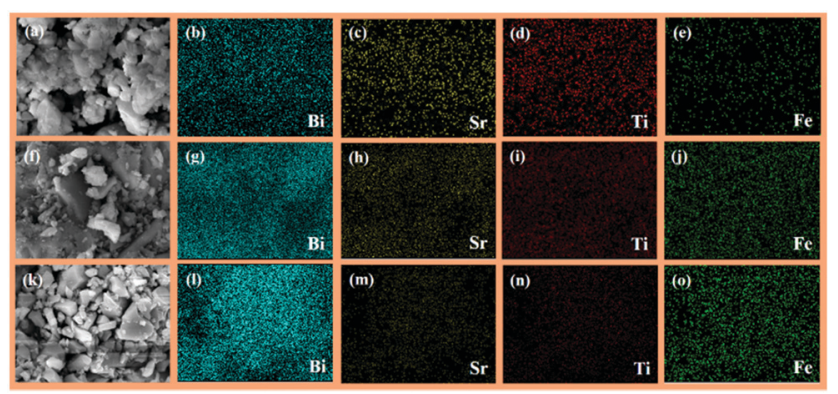

Fig. 3 SEM images and corresponding EDS elemental maps for $\mathrm{Bi}_{5.25} \mathrm{Sr}_{0.75^{-}}$ $\mathrm{Ti}_{3.75} \mathrm{Fe}_{1.25} \mathrm{O}_{18}(\mathrm{a}-\mathrm{e}), \mathrm{Bi}_{5.5} \mathrm{Sr}_{0.5} \mathrm{Ti}_{3.5} \mathrm{Fe}_{1.5} \mathrm{O}_{18}$ (f-j) and $\mathrm{Bi}_{5.75} \mathrm{Sr}_{0.25} \mathrm{Ti}_{3.25} \mathrm{Fe}_{1.75} \mathrm{O}_{18}$ $(\mathrm{k}-\mathrm{O})$.

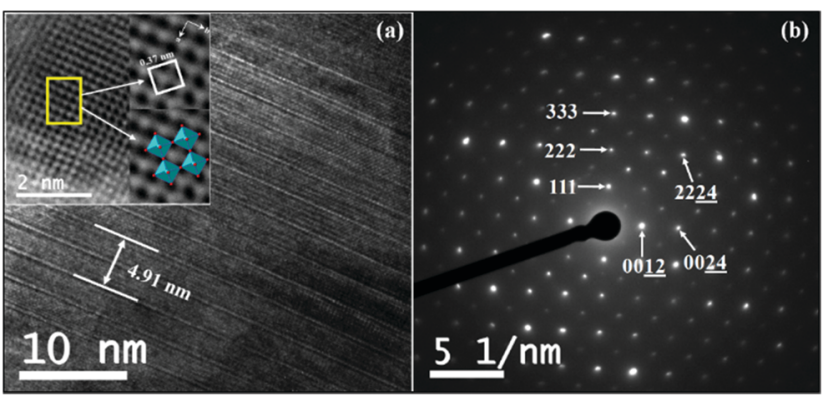

Fig. 4 (a) HR-TEM image showing periodic lattice fringes in the $c$-direction (the inset shows lattice periodicity in the ab-plane) and (b) SAED pattern of $\mathrm{Bi}_{5.75} \mathrm{Sr}_{0.25} \mathrm{Ti}_{3.25} \mathrm{Fe}_{1.75} \mathrm{O}_{18}$.

absorption at the lower wavelength side, while the higher wavelength edge is more stretched. This is attributed to the electronic band structure of the compounds. It is presumed that the valence band (VB) states of these Aurivillius phases are made up of Fe (3d), Bi (6s) and O (2p) hybridized states, while the conduction band (CB) states are mostly made up of Ti (3d) and Bi (6p) states. ${ }^{60}$ The extension of absorption edges in the visible region is clear with increasing amount of $\mathrm{Fe}$ in the compounds and this corresponds to the build-up of Fe (3d) states on the top of valence band. Subsequently, the higher energy edge is also red-shifted consistent with the decreasing trend of band gaps in the compounds. Band gap energies of these compounds are determined by the Tauc plots (see Table S2 and Fig. S3 in the ESI $\dagger$ ). The plots suggested an indirect band gap transition as $(\alpha h \nu)^{1 / n} v s$. $h \nu$ was better fitted for $n=2$. It can be claimed that the UV-vis absorption features do not change significantly in the series $\mathrm{Fe}_{1.25}$ to $\mathrm{Fe}_{1.75}$, although more $\mathrm{Fe}$ (3d) states build up with increased density of states (DOS) contribution near the top of the valence band.

\section{PL and FLS analysis}

The PL spectra of the compounds (Fig. 5) recorded at an excitation wavelength of $355 \mathrm{~nm}$ give a broad emission (425$510 \mathrm{~nm}$ ) that peaks around $470 \mathrm{~nm}$. Intensity of PL spectra gives indirect information about charge carrier separation in $\mathrm{Bi}_{6-x} \mathrm{Sr}_{x} \mathrm{Ti}_{3+x} \mathrm{Fe}_{2-x} \mathrm{O}_{18}(x=1-0)$. A higher PL intensity suggests greater radiative $\mathrm{e}^{-}-\mathrm{h}^{+}$recombination leaving fewer charge separated carriers ${ }^{61}$ that can take part in the photocatalytic activity. Thus, one can expect photocatalytic activity in the order,
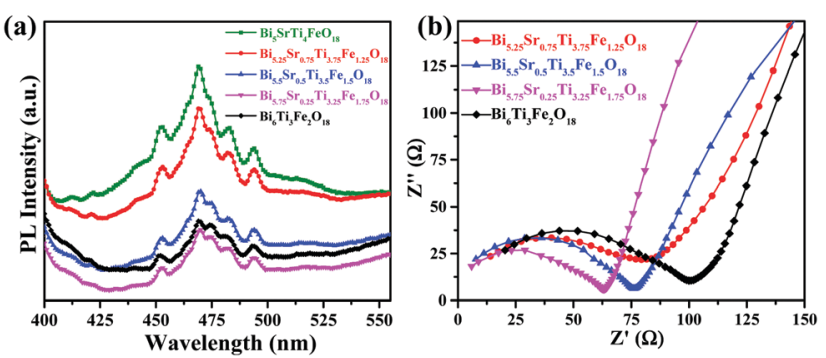

Fig. 5 (a) PL spectra $\left(\lambda_{\text {ex }}=355 \mathrm{~nm}\right)$ of $\mathrm{Bi}_{6-x} \mathrm{Sr}_{x} \mathrm{Ti}_{3+x} \mathrm{Fe}_{2-x} \mathrm{O}_{18}(x=1,0.75$, $0.5,0.25$ and 0.0$)$. (b) EIS Nyquist plots of $\mathrm{Bi}_{6-x} \mathrm{Sr}_{x} \mathrm{Ti}_{3+x} \mathrm{Fe}_{2-x} \mathrm{O}_{18}(x=0.75$, $0.5,0.25$ and 0.0 ). 
$\mathrm{Bi}_{5.75} \mathrm{Sr}_{0.25} \mathrm{Ti}_{3.25} \mathrm{Fe}_{1.75} \mathrm{O}_{18}>\mathrm{Bi}_{6} \mathrm{Ti}_{3} \mathrm{Fe}_{2} \mathrm{O}_{18}>\mathrm{Bi}_{5.5} \mathrm{Sr}_{0.5} \mathrm{Ti}_{3.5^{-}}$ $\mathrm{Fe}_{1.5} \mathrm{O}_{18}>\mathrm{Bi}_{5.25} \mathrm{Sr}_{0.75} \mathrm{Ti}_{3.75} \mathrm{Fe}_{1.25} \mathrm{O}_{18}>\mathrm{Bi}_{5} \mathrm{SrTi}_{4} \mathrm{FeO}_{18}$, by comparing the PL intensities (Fig. 5a) although the final activity order is different depending on the dominance of other competing factors.

FLS analysis tells us about the lifetime of photogenerated charge species. The radiative lifetimes and their percentage contributions are obtained by fitting the FLS decay spectra (Fig. 6). An enhancement in decay lifetime is observed for $\mathrm{Bi}_{5.75} \mathrm{Sr}_{0.25} \mathrm{Ti}_{3.25} \mathrm{Fe}_{1.75} \mathrm{O}_{18}$ with a relatively stretched exponential decay of fluorescence intensity. The average lifetime $\left(\tau_{\text {avg }}\right)$ is calculated using a three exponential function using the following formula: ${ }^{62}$

$$
\tau_{\mathrm{avg}}=\sum_{i=1}^{3} A_{i} B_{i}^{2} / \sum_{i=1}^{3} A_{i} B_{i}
$$

where, $\tau_{\text {avg }}$ is the average lifetime and $A_{i}$ and $B_{i}$ are the amplitude and radiative lifetimes, respectively. The $\tau_{\text {avg }}$ values of $\mathrm{Bi}_{5.25} \mathrm{Sr}_{0.75^{-}}$ $\mathrm{Ti}_{3.75} \mathrm{Fe}_{1.25} \mathrm{O}_{18}, \mathrm{Bi}_{5.5} \mathrm{Sr}_{0.5} \mathrm{Ti}_{3.5} \mathrm{Fe}_{1.5} \mathrm{O}_{18}$ and $\mathrm{Bi}_{5.75} \mathrm{Sr}_{0.25} \mathrm{Ti}_{3.25} \mathrm{Fe}_{1.75} \mathrm{O}_{18}$ are 4.66, 6.29 and $10.22 \mathrm{~ns}$, respectively. The higher average fluorescence lifetime in $\mathrm{Bi}_{5.75} \mathrm{Sr}_{0.25} \mathrm{Ti}_{3.25} \mathrm{Fe}_{1.75} \mathrm{O}_{18}$ indicates efficient separation of charge carriers and suppression of the $\mathrm{e}^{-}-\mathrm{h}^{+}$ recombination process leading eventually to higher photocatalytic activity in the compound (see the Photocatalytic activity section).

\section{EIS analysis}

EIS measurements are carried out to study the electrical properties at the interface such as charge transfer resistance $\left(R_{\mathrm{CT}}\right)$ to the flow of photogenerated charge carriers. The corresponding EIS Nyquist plots (Fig. 5b) of the compounds give a semicircle in the higher frequency side signifying the $R_{\mathrm{CT}}$ and a tail at lower frequency range implies the Warburg impedance contribution. $\mathrm{Bi}_{5.75} \mathrm{Sr}_{0.25} \mathrm{Ti}_{3.25} \mathrm{Fe}_{1.75} \mathrm{O}_{18}$ indicate a smaller semicircle as compared to others implying a smaller $R_{\mathrm{CT}}$. The estimated $R_{\mathrm{CT}}$ values for $\mathrm{Bi}_{5.75} \mathrm{Sr}_{0.25} \mathrm{Ti}_{3.25} \mathrm{Fe}_{1.75} \mathrm{O}_{18}, \quad \mathrm{Bi}_{5.5} \mathrm{Sr}_{0.5} \mathrm{Ti}_{3.5} \mathrm{Fe}_{1.5} \mathrm{O}_{18}, \quad \mathrm{Bi}_{5.25} \mathrm{Sr}_{0.75} \mathrm{Ti}_{3.75}$ $\mathrm{Fe}_{1.25} \mathrm{O}_{18}$ and $\mathrm{Bi}_{6} \mathrm{Ti}_{3} \mathrm{Fe}_{2} \mathrm{O}_{18}$ are 58.7, 68.3, 79.9 and 83.6 $\Omega$, respectively. This would help the easy transfer of electron and holes at $\mathrm{Bi}_{5.75} \mathrm{Sr}_{0.25} \mathrm{Ti}_{3.25} \mathrm{Fe}_{1.75} \mathrm{O}_{18}$ semiconductor surfaces, implying improved activity compared to other compounds with higher $R_{\mathrm{CT}}$.

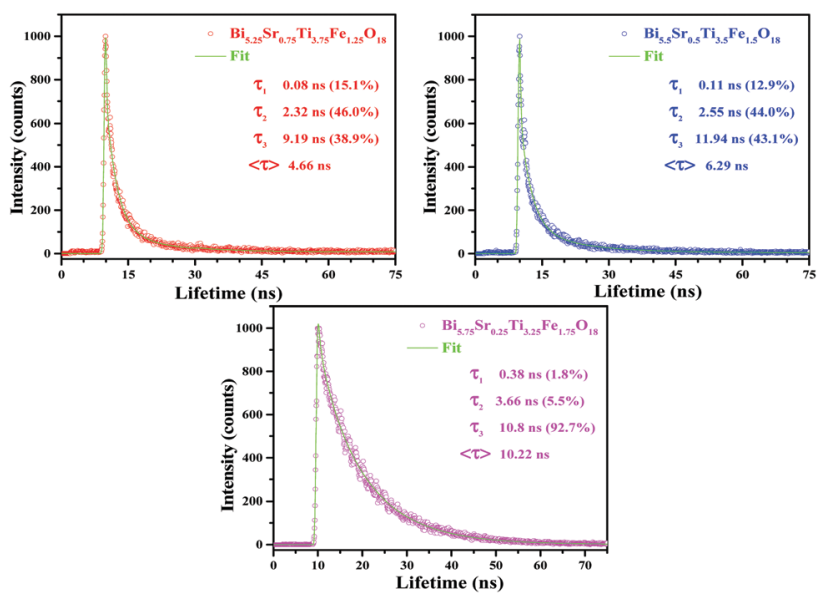

Fig. 6 FLS spectra of $\mathrm{Bi}_{6-x} \mathrm{Sr}_{x} \mathrm{Ti}_{3+x} \mathrm{Fe}_{2-x} \mathrm{O}_{18}(x=0.75,0.50$ and 0.25$)$.
The EIS analysis of $\mathrm{Bi}_{5.75} \mathrm{Sr}_{0.25} \mathrm{Ti}_{3.25} \mathrm{Fe}_{1.75} \mathrm{O}_{18}$ under light illumination and an external bias is carried out to understand the actual resistance to charge-transfer and $\mathrm{e}^{-}-\mathrm{h}^{+}$pair recombination dynamics during photocatalysis. We observed a lower $R_{\mathrm{CT}}$ at $1 \mathrm{~V}$ applied bias compared to that at $0 \mathrm{~V}$ under simulated solar illumination (see Fig. S4a in the ESI $\dagger$ ). This suggests that the compound would show higher photocatalytic activity under the applied bias. The effect of applied voltage on the photocatalytic activity is studied and discussed in the next section. The charge transfer resistance $\left(R_{\mathrm{CT}}\right)$, double layer capacitance $\left(C_{\mathrm{dl}}\right)$ and Warburg parameters of all the samples under external bias have been estimated for comparison by fitting the impedance data using a conventional $R-C$ circuit and Warburg impedance (Fig. S5-S7 and Table S6 in the ESI $\dagger$ ).

\section{Photocatalytic activity}

Photocatalytic activities of the semiconductor photocatalysts are estimated by way of dye degradation. For this, we have selected aqueous solutions of RhB dye as a model system for photocatalysis and the quantity of catalyst used was determined by dosage optimization results (Fig. S1, ESI $\dagger$ ). The compounds, $\mathrm{Bi}_{6-x} \mathrm{Sr}_{x} \mathrm{Ti}_{3+x} \mathrm{Fe}_{2-x} \mathrm{O}_{18}(x=0.75,0.5,0.25)$, showed very efficient photocatalytic activity toward complete degradation of RhB at pH 2 within 20 to 25 minutes of sunlight irradiation (Fig. 7a). The UV-vis absorption data (Fig. S8, ESI $\dagger$ ) also show clear-cut fast kinetics for $\mathrm{Bi}_{5.75} \mathrm{Sr}_{0.25} \mathrm{Ti}_{3.25} \mathrm{Fe}_{1.75} \mathrm{O}_{18}$, whereas the compounds with $\mathrm{Fe}_{1.25}$ and $\mathrm{Fe}_{1.5}$ show similar kinetics. Moreover, there is negligible or no degradation of $\mathrm{RhB}$ at $\mathrm{pH} 7$ and 11 (Fig. S9, ESI $\dagger$ ) and no self-degradation or photolysis of the dye in the sunlight at any of the $\mathrm{pH}$ investigated (Fig. 7 and Fig. S9, ESI $\dagger$ ).

The kinetics of the photocatalytic RhB degradation over $\mathrm{Bi}_{6-x} \mathrm{Sr}_{x} \mathrm{Ti}_{3+x} \mathrm{Fe}_{2-x} \mathrm{O}_{18}(x=0.75,0.50,0.25)$ was fitted with a Langmuir-Hinshelwood rate expression. ${ }^{42}$

$$
\ln \left(C_{0} / C\right)=k t
$$

where, $C_{0}$ is the initial dye concentration, $C$ is the dye concentration at time $t$ and $k$ is the pseudo-first-order rate constant. The linearity of $\ln \left(C_{0} / C\right) v s$. irradiation time $(t)$ plot indicates that photocatalytic $\mathrm{RhB}$ degradation over $\mathrm{Bi}_{6-x} \mathrm{Sr}_{x} \mathrm{Ti}_{3+x} \mathrm{Fe}_{2-x} \mathrm{O}_{18}$ $(x=0.75,0.5$, and 0.25$)$ follows a first-order kinetics. The degradation rate constants $(k)$ calculated from the slope of $\ln \left(C_{0} / C\right)$ versus $t$ plot (Fig. 7b) shows the highest value $\left(k=0.2170 \mathrm{~min}^{-1}\right)$ for $\mathrm{Bi}_{5.75} \mathrm{Sr}_{0.25} \mathrm{Ti}_{3.25} \mathrm{Fe}_{1.75} \mathrm{O}_{18}$ and while decreases in the order
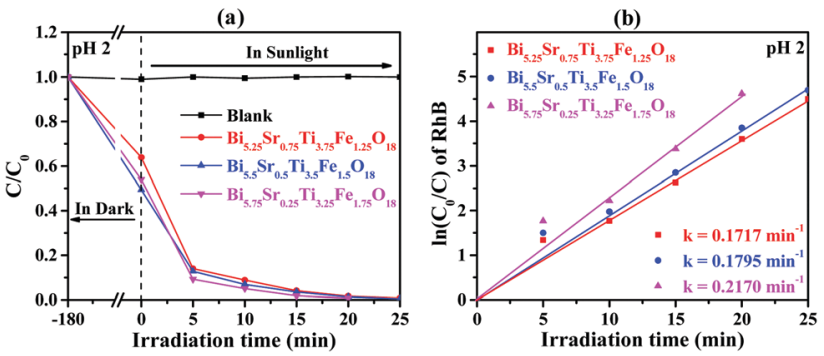

Fig. 7 (a) $\mathrm{C}_{0} / \mathrm{C}$ vs. irradiation time for $\mathrm{RhB}$ at $\mathrm{pH} 2$ and (b) corresponding $\ln \left(C_{0} / C\right)$ as a function of irradiation time over $\mathrm{Bi}_{6-x} \mathrm{Sr}_{x} \mathrm{Ti}_{3+x} \mathrm{Fe}_{2-x} \mathrm{O}_{18}$ $(x=0.75,0.5$, and 0.25$)$ 
$\mathrm{Bi}_{5.75} \mathrm{Sr}_{0.25} \mathrm{Ti}_{3.25} \mathrm{Fe}_{1.75} \mathrm{O}_{18}>\mathrm{Bi}_{5.5} \mathrm{Sr}_{0.5} \mathrm{Ti}_{3.5} \mathrm{Fe}_{1.5} \mathrm{O}_{18} \quad(k=$ $\left.0.1795 \mathrm{~min}^{-1}\right)>\mathrm{Bi}_{5.25} \mathrm{Sr}_{0.75} \mathrm{Ti}_{3.75} \mathrm{Fe}_{1.25} \mathrm{O}_{18}\left(k=0.1717 \mathrm{~min}^{-1}\right)$.

The effect of applied voltage on the photocatalytic activity is studied by the degradation of $\mathrm{RhB}$ at 0 and $1 \mathrm{~V}$ applied bias for five minutes under simulated solar light. At $1 \mathrm{~V}$ bias, $\mathrm{Bi}_{5.75} \mathrm{Sr}_{0.25} \mathrm{Ti}_{3.25} \mathrm{Fe}_{1.75} \mathrm{O}_{18}$ degrades $\sim 64 \%$ of dye while only $\sim 44 \%$ dye is degraded at $0 \mathrm{~V}$ after five minutes of irradiation (see Fig. S4b in the ESI $\dagger$ ). The enhanced activity is correlated with the additional band bending effects (see later section) in the presence of an external electric field, in addition to the already existing internal electric fields in the compounds. It is believed that under the external electric field the ferroelectric domains and local surface dipoles in powder samples preferentially orient in the direction of the field improving the carrier separation efficiency. Moreover, the enhanced thermal barrier due to an upward band bending also reduces the chances of $\mathrm{e}^{-}-\mathrm{h}^{+}$recombination.

\section{Detection of reactive species}

A series of scavenger tests were performed in the presence of ammonium oxalate $\left(\mathrm{AO}, \mathrm{h}^{+}\right.$scavenger), benzoquinone (BQ, $\mathrm{O}_{2}{ }^{\bullet-}$ scavenger) and tertiary butyl alcohol $\left(t-\mathrm{BuOH},{ }^{\bullet} \mathrm{OH}\right.$ scavenger $)$ to ascertain the reactive species responsible for the photocatalytic RhB degradation over $\mathrm{Bi}_{5.75} \mathrm{Sr}_{0.25} \mathrm{Ti}_{3.25} \mathrm{Fe}_{1.75} \mathrm{O}_{18}$. The effect of these scavengers on the RhB degradation efficiency of $\mathrm{Bi}_{5.75} \mathrm{Sr}_{0.25}$ $\mathrm{Ti}_{3.25} \mathrm{Fe}_{1.75} \mathrm{O}_{18}$ at $\mathrm{pH} 2$ is shown in Fig. 8. Addition of AO to the dye-photocatalyst suspension greatly reduces the photodegradation of RhB as compared to that in the absence of any scavenger. Moreover, addition of $\mathrm{BQ}$ to the dye-photocatalyst suspension causes a significant reduction in $\mathrm{RhB}$ degradation, while the addition of $t$-BuOH to the dye-photocatalyst suspension caused reduction in photodegradation only to a small extent at this $\mathrm{pH}$. Thus, the above results indicate that $\mathrm{h}^{+}$and $\mathrm{O}_{2}{ }^{\bullet-}$ are key active species involved in the photocatalytic degradation of RhB over $\mathrm{Bi}_{5.75} \mathrm{Sr}_{0.25} \mathrm{Ti}_{3.25} \mathrm{Fe}_{1.75} \mathrm{O}_{18}$ in the acidic medium and ${ }^{\bullet} \mathrm{OH}$ plays a negligible role in the degradation.

\section{Stability and reusability studies}

Whether the Aurivillius photocatalysts are reusable and stable at ambient conditions or not is important for their repeated use.

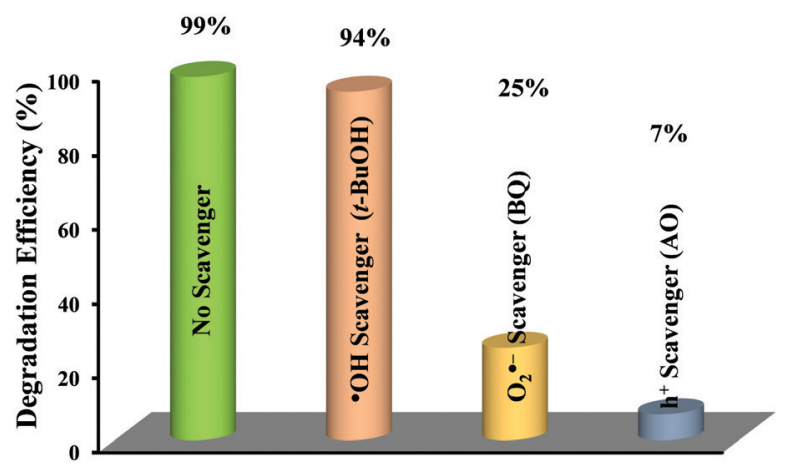

At 20 min irradiation

Fig. 8 Effect of different scavengers on the degradation efficiency of RhB over $\mathrm{Bi}_{5.75} \mathrm{Sr}_{0.25} \mathrm{Ti}_{3.25} \mathrm{Fe}_{1.75} \mathrm{O}_{18}$ under sunlight irradiation.
The reusability study of $\mathrm{Bi}_{5.75} \mathrm{Sr}_{0.25} \mathrm{Ti}_{3.25} \mathrm{Fe}_{1.75} \mathrm{O}_{18}$ for five successive cycles (Fig. S10a, ESI $\dagger$ ) shows $\sim 98 \%$ of dye degradation even in the 5 th cycle almost without any change in its ability. The slight decrease $(\sim 2 \%)$ in the activity is due to the inevitable loss of catalysts during the experiment. The stability of $\mathrm{Bi}_{5.75} \mathrm{Sr}_{0.25} \mathrm{Ti}_{3.25} \mathrm{Fe}_{1.75} \mathrm{O}_{18}$ is also assessed by PXRD, HR-TEM and XPS analysis of the compound recovered after the 5th cycle. PXRD patterns (Fig. S10b, ESI $\dagger$ ) show retention of phase purity with sharp peaks affirming the unchanged crystallinity of the compound. The HR-TEM analysis (Fig. S10c, ESI $\dagger$ ) of postcatalytic samples also reaffirmed preservation of good crystallinity without any expansion or contraction in lattice parameters, as the distances between fringes remained intact before and after the photocatalysis. Similarly, post-catalytic PXRD of the $\mathrm{Fe}_{1.25}$ and $\mathrm{Fe}_{1.5}$ analogues also demonstrated good catalyst stability under the reaction conditions (Fig. S11, ESI $\dagger$ ).

Fig. 9 represents the XPS data of $\mathrm{Bi}_{5.75} \mathrm{Sr}_{0.25} \mathrm{Ti}_{3.25} \mathrm{Fe}_{1.75} \mathrm{O}_{18}$ recorded before and after the photocatalytic studies. While the survey scan shows surface presence of all the elements, the high-resolution elemental scan shows that all the elements in $\mathrm{Bi}_{5.75} \mathrm{Sr}_{0.25} \mathrm{Ti}_{3.25} \mathrm{Fe}_{1.75} \mathrm{O}_{18}$ are chemically stable. High-resolution XPS of Bi $4 \mathrm{f}$ (Fig. 9b) show spin-orbit coupled $\mathrm{Bi} 4 \mathrm{f}_{7 / 2}$ and $\mathrm{Bi}$ $4 \mathrm{f}_{5 / 2}$ states at the binding energy (B.E.) of 158.8 and $164.1 \mathrm{eV}$, respectively, confirming the presence of $\mathrm{Bi}$ in +3 oxidation state. Similarly, Ti $2 \mathrm{p}_{3 / 2}$ and $\mathrm{Ti} 2 \mathrm{p}_{1 / 2}$ states with B.E. of 457.7 and $462.8 \mathrm{eV}$ (Fig. 9d) confirm the presence of $\mathrm{Ti}^{4+}$. In the same region, an additional peak of $\mathrm{Bi} 4 \mathrm{~d}_{3 / 2}$ at $465.6 \mathrm{eV}$ is also seen consistent with the $\mathrm{Bi}^{3+}$ state. In the Fe $2 p$ spectra (Fig. 9e), the spin-orbit coupled states, $2 \mathrm{p}_{3 / 2}$ and $2 \mathrm{p}_{1 / 2}$, appear at the B.E. of 710.2 and $723.7 \mathrm{eV}$, respectively, which corroborates well with the presence of $\mathrm{Fe}^{3+}$ in the compound before and after photocatalysis. Likewise, Sr 3d XPS (Fig. 9c) also show spin-orbit coupled doublet of $3 \mathrm{~d}_{5 / 2}$ and $3 \mathrm{~d}_{3 / 2}$ states corresponding to the presence of $\mathrm{Sr}^{2+}$.

The O 1s XPS peaks at 529.3-529.5 and 531.1 eV (Fig. 9f) normally correspond to the lattice and adsorbed or hydroxylated oxygens, respectively. While the intensity of lattice oxygen peaks appeared to remain same before and after the photocatalysis, the peak intensity at $531.1 \mathrm{eV}$ appeared to have increased slightly. This generally corresponds to an increase in adsorbed oxygen or hydroxylated species and it is natural to have this
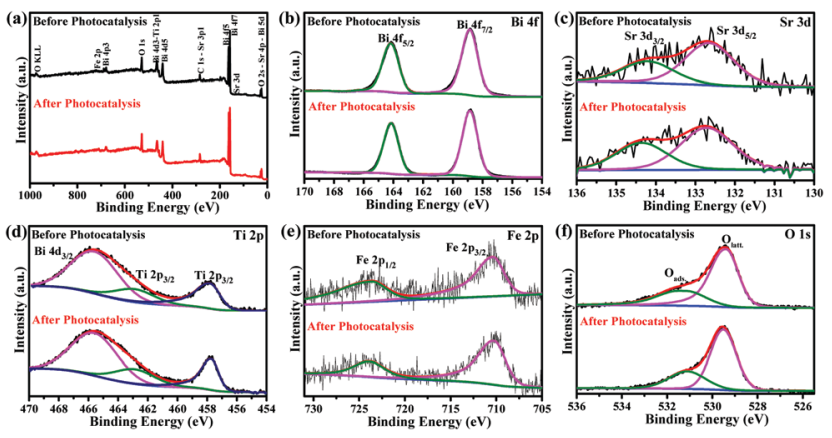

Fig. 9 (a) Survey and (b-f) high-resolution XPS of Bi $4 f(b), S r 3 d$ (c), Ti $2 p$ (d), Fe $2 \mathrm{p}(\mathrm{e})$ and $\mathrm{O}$ 1s (f) of $\mathrm{Bi}_{5.75} \mathrm{Sr}_{0.25} \mathrm{Ti}_{3.25} \mathrm{Fe}_{1.75} \mathrm{O}_{18}$, before and after five cycles of photocatalytic RhB degradation at $\mathrm{pH} 2$. 
increase due to the continuous exposure of the compound to the acidic aqueous atmosphere in several catalytic cycles. Considering the harsh environment at $\mathrm{pH}$ 2, post-catalytic PXRD, HR-TEM and XPS studies indicate that the Aurivillius compounds are robust under the photocatalytic reaction condition and are stable to photoleaching or photocorrosion and phase decomposition. Moreover, the intact chemical state of the transition element (Fe in this case) indicates the pure photocatalytic nature of the dye degradation process and the absence of any redox catalytic processes during degradation.

\section{Adsorption}

It is well established and proved from earlier findings that adsorption of dye at the catalyst surface plays a vital role in photocatalytic degradation. Hence to determine the effect of adsorption on the photocatalytic activity, RhB adsorption tests are performed over $\mathrm{Bi}_{6-x} \mathrm{Sr}_{x} \mathrm{Ti}_{3+x} \mathrm{Fe}_{2-x} \mathrm{O}_{18}(x=0.75,0.5$, and 0.25) under acidic, neutral and alkaline $\mathrm{pH}$. The adsorption results show the highest adsorption at acidic $\mathrm{pH}$ (Fig. 10a) and order varies as $\mathrm{Bi}_{5.5} \mathrm{Sr}_{0.5} \mathrm{Ti}_{3.5} \mathrm{Fe}_{1.5} \mathrm{O}_{18}>\mathrm{Bi}_{5.75} \mathrm{Sr}_{0.25} \mathrm{Ti}_{3.25} \mathrm{Fe}_{1.75} \mathrm{O}_{18}>$ $\mathrm{Bi}_{5.25} \mathrm{Sr}_{0.75} \mathrm{Ti}_{3.75} \mathrm{Fe}_{1.25} \mathrm{O}_{18}$. The photocatalytic activity order of the compounds, however, did not follow the adsorption order, in contrast to earlier reports. This brings out the important role of other competing factors in determining the overall activity of these closely related Aurivillius solid-solution phases.

\section{$\zeta$-Potential study}

As the surface charge of catalysts is an important factor for dye adsorption on the catalyst, the $\zeta$-potential is measured at different $\mathrm{pH}$ for the compounds (Fig. 10b). The highest negative $\zeta$-potential for the compounds is observed at alkaline $\mathrm{pH}$ and it gradually decreases from more negative to less negative and finally reaches positive values in the acidic medium ( $\mathrm{pH} 2)$. The following trend is due to the action of $\mathrm{H}^{+}$and $\mathrm{OH}^{-}$ions on the surface oxygen or hydroxyl groups attached to the surface of the oxide catalysts. ${ }^{5}$ The potential of zero charge (pzc) arises at a lower $\mathrm{pH}$ for $\mathrm{Fe}_{1.75}$ as compared to those of others. This indicates a larger intrinsic negative surface charge.

\section{Mechanism of photocatalytic activity}

The mechanism of photocatalytic degradation can be interpreted from the energy level diagram (ELD) presented in Fig. 11. All the potentials shown in Fig. 11 are with reference to normal hydrogen electrode (NHE) potentials. The flat-band potential $\left(E_{\mathrm{fb}}\right)$ of

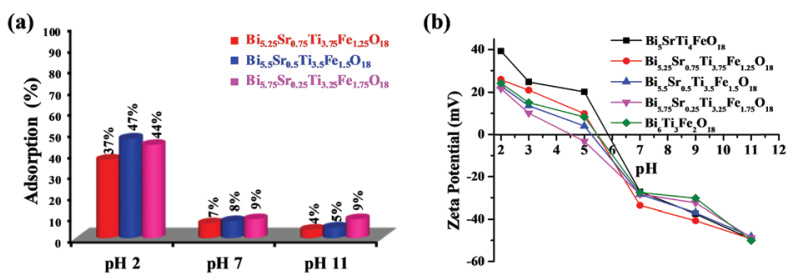

Fig. 10 (a) Adsorption percentage of $\mathrm{RhB}$ dye over of $\mathrm{Bi}_{6-x} \mathrm{Sr}_{x} \mathrm{Ti}_{3+x} \mathrm{Fe}_{2-x} \mathrm{O}_{18}$ $(x=0.75,0.50 \& 0.25)$ at different $\mathrm{pH}$ values. (b) $\zeta$-Potential of $\mathrm{Bi}_{6-x} \mathrm{Sr}_{x^{-}}$ $\mathrm{Ti}_{3+x} \mathrm{Fe}_{2-x} \mathrm{O}_{18}(x=1-0)$ catalysts at different $\mathrm{pH}$.
$-0.511 \mathrm{~V}$ vs. SCE calculated for $\mathrm{Bi}_{5.75} \mathrm{Sr}_{0.25} \mathrm{Ti}_{3.25} \mathrm{Fe}_{1.75} \mathrm{O}_{18}$ with help of Mott-Schottky plots (see Fig. S12 in ESI $\dagger$ ) corresponds to $-0.269 \mathrm{~V}$ w.r.t. NHE. As $E_{\mathrm{fb}}$ is very close to the bottom of $E_{\mathrm{CB}}(1)$ in n-type semiconductors, $E_{\mathrm{fb}}$ is considered as $E_{\mathrm{CB}}(1)(-0.269 \mathrm{~V})$ and $E_{\mathrm{VB}}(1.811 \mathrm{~V})$ is calculated using the band gap $(2.08 \mathrm{eV})$ from the expression given below.

$$
E_{\mathrm{VB}}=E_{\mathrm{CB}}+E_{\mathrm{g}}
$$

Similarly, the position of the higher energy edge, $E_{\mathrm{CB}}(2)$ $(-0.819 \mathrm{~V})$ is calculated using the energy of higher band edge $(2.62 \mathrm{eV})$. The positions for $\mathrm{CB}$ and $\mathrm{VB}$ edges for $\mathrm{Bi}_{6-x} \mathrm{Sr}_{x} \mathrm{Ti}_{3+x} \mathrm{Fe}_{2-x} \mathrm{O}_{18}(x=0.0,0.75,0.5$ and 0.25$)$ calculated using the flat band potentials (see Table S7 in ESI $\dagger$ ) are summarized in Table 1 and are shown in Fig. S13 of the ESI. $\dagger$ It is evident from the ELD (Fig. 11) that $E_{\mathrm{CB}}(2)$ of $\mathrm{Bi}_{5.75} \mathrm{Sr}_{0.25} \mathrm{Ti}_{3.25} \mathrm{Fe}_{1.75} \mathrm{O}_{18}(-0.82 \mathrm{~V}$ vs. NHE $)$ is suitable for generation of the $\mathrm{O}_{2}{ }^{--}\left(E_{\mathrm{O}_{2} / \mathrm{O}_{2}{ }^{--}}=-0.33 \mathrm{~V} v s\right.$. NHE $),{ }^{63}$ therefore, the photoelectrons from $E_{\mathrm{CB}}(2)$ could be transferred to adsorbed oxygens to produce $\mathrm{O}_{2}{ }^{\bullet-}$ radicals. In addition, the holes generated in VB can also be transported directly to HOMO of adsorbed RhB molecules and thereby degrade it. However, the VB potential $(+1.81 \mathrm{~V} v s$. NHE) is not suitable for generation of ${ }^{\bullet} \mathrm{OH}$ radicals $\left(E \cdot \bullet^{\circ} \mathrm{OH} / \mathrm{H}_{2} \mathrm{O}=+2.68 \mathrm{~V} v s \text {. } \mathrm{NHE}\right)^{61}$ by

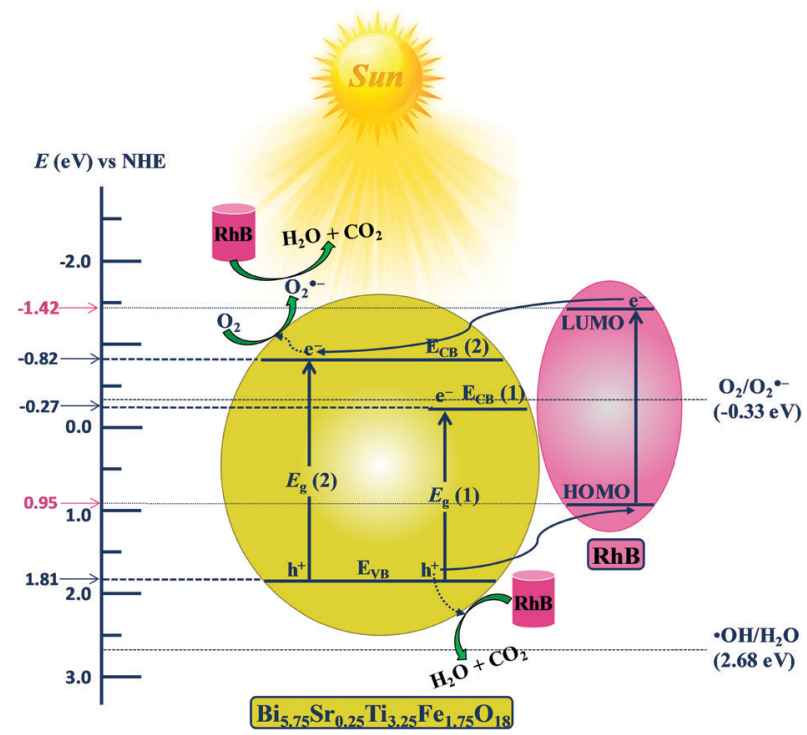

Fig. 11 Schematic energy level diagram of $\mathrm{Bi}_{5.75} \mathrm{Sr}_{0.25} \mathrm{Ti}_{3.25} \mathrm{Fe}_{1.75} \mathrm{O}_{18}$ with respect to the potential of ${ }^{\bullet} \mathrm{OH} / \mathrm{H}_{2} \mathrm{O}\left(\mathrm{E}^{\bullet} \mathrm{OH} / \mathrm{H}_{2} \mathrm{O}\right), \mathrm{O}_{2} / \mathrm{O}_{2} \cdot{ }^{--}\left(E_{\mathrm{O}_{2} / \mathrm{O}_{2}}{ }^{-}\right)$and the HOMO-LUMO levels of RhB (NHE scale).

Table 1 Calculated values of valence and conduction band positions for $\mathrm{Bi}_{6-x} \mathrm{Sr}_{x} \mathrm{Ti}_{3+x} \mathrm{Fe}_{2-x} \mathrm{O}_{18}(x=0.75,0.5,0.25,0.0)$

\begin{tabular}{|c|c|c|c|}
\hline \multirow[b]{2}{*}{ Compound } & \multirow{2}{*}{$\begin{array}{l}\text { Valence band, } \\
E_{\mathrm{VB}}(\mathrm{V})\end{array}$} & \multicolumn{2}{|c|}{ Conduction band } \\
\hline & & $E_{\mathrm{CB}}(1)(\mathrm{V})$ & $E_{\mathrm{CB}}(2)(\mathrm{V})$ \\
\hline $\mathrm{Bi}_{5.25} \mathrm{Sr}_{0.75} \mathrm{Ti}_{3.75} \mathrm{Fe}_{1.25} \mathrm{O}_{18}$ & 1.797 & -0.333 & -0.863 \\
\hline $\mathrm{Bi}_{5.5} \mathrm{Sr}_{0.5} \mathrm{Ti}_{3.5} \mathrm{Fe}_{1.5} \mathrm{O}_{18}$ & 1.954 & -0.156 & -0.686 \\
\hline $\mathrm{Bi}_{5.75} \mathrm{Sr}_{0.25} \mathrm{Ti}_{3.25} \mathrm{Fe}_{1.75} \mathrm{O}_{18}$ & 1.811 & -0.269 & -0.819 \\
\hline $\mathrm{Bi}_{6} \mathrm{Ti}_{3} \mathrm{Fe}_{2} \mathrm{O}_{18}$ & 1.850 & -0.230 & -0.809 \\
\hline
\end{tabular}


oxidizing $\mathrm{H}_{2} \mathrm{O}$ molecules. The above findings are consistent with the scavenger studies showing $\mathrm{O}_{2}{ }^{--}$and $\mathrm{h}^{+}$as the major reactive species responsible for the $\mathrm{RhB}$ degradation. The overall mechanism of $\mathrm{O}_{2}{ }^{\bullet-}$ and $\mathrm{h}^{+}$mediated $\mathrm{RhB}$ degradation do not vary across the series of compounds given the close and unaltered positioning of their VB and CB edges.

The role of the crystal structure in the photocatalytic activity of the Aurivillius phase perovskites is evaluated. The catalyst surface charge can be influenced by an unsymmetrical bond polarization due to its non-centrosymmetric crystal structure (space group, $F 2 \mathrm{~mm}$ ). The octahedral distortion parameter ${ }^{64,65}$ $\left(\Delta_{\mathrm{o}}\right)$ of the compounds are calculated for all the $\mathrm{Fe} / \mathrm{TiO}_{6}$ octahedra to analyse the role of structural distortion on the RhB adsorption on the catalyst surface. The octahedral distortion parameter is defined as, $\Delta_{\mathrm{o}}=\frac{1}{6} \sum_{i=1}^{6}\left[\left(l_{i}-\bar{l}\right) / \bar{l}\right]^{2}$ where, the sum is over six bonds of the octahedra, $\bar{l}$ is the average bond distance and $l_{i}$ are individual bond distances. The structure with central, pre-terminal and terminal octahedra along with their bond distances for $\mathrm{Bi}_{6-x} \mathrm{Sr}_{x} \mathrm{Ti}_{3+x} \mathrm{Fe}_{2-x} \mathrm{O}_{18}$ $(x=0.75,0.5$, and 0.25$)$ are shown in Fig. S14 (ESI $\dagger)$ and the calculated $\Delta_{\mathrm{o}}$ values are given in Table S8 of the ESI. $\dagger$

The highest $\Delta_{\mathrm{o}}$ value of 0.0115 is obtained for the terminal octahedra of $\mathrm{Bi}_{5.5} \mathrm{Sr}_{0.5} \mathrm{Ti}_{3.5} \mathrm{Fe}_{1.5} \mathrm{O}_{18}$, while relatively smaller $\Delta_{\mathrm{o}}$ values of 0.0106 and 0.0076 are found for $\mathrm{Bi}_{5.75} \mathrm{Sr}_{0.25} \mathrm{Ti}_{3.25} \mathrm{Fe}_{1.75} \mathrm{O}_{18}$ and $\mathrm{Bi}_{5.25} \mathrm{Sr}_{0.75} \mathrm{Ti}_{3.75} \mathrm{Fe}_{1.25} \mathrm{O}_{18}$, respectively. As reported in previous studies that the $\Delta_{\mathrm{o}}$ values gradually decreases in the order, terminal $>$ pre-terminal $>$ central octahedral layer for the fivelayer Aurivillius phase perovskites in a given series of compounds, is consistent with the second-order Jahn-Teller (SOJT) distortion of $\mathrm{d}^{0}$ cations in these compounds. ${ }^{66-68}$ The increase in local octahedral distortion enhances the extent of adsorption of dye molecules on the catalyst surface. Furthermore, the axial distortion parameter, defined as, $\Delta_{\mathrm{ax}}=\frac{1}{2} \sum_{i=1}^{2}\left[\left(l_{i}-\bar{l}\right) / \bar{l}\right]^{2}$, where the $l_{i}$ are the two axial bond lengths in an octahedra parallel to the crystallographic $c$-axis, is also calculated (Table S8, ESI $\dagger$ ). Interestingly, the variation of $\Delta_{\mathrm{ax}}$ across the octahedral layers for any given compound also follows the order of distortion similar to $\Delta_{\mathrm{o}}$, being highest for the terminal and lowest in the central octahedral layer. It is shown that the crystal chemical correlation can be exploited in the catalytic activity enhancement by designing oxides with increased axial octahedral distortion in a homologous series, wherein the frontier band structure and photoluminescence properties largely remain unaffected due to compositional modifications at the A-sites of the perovskites largely by alkali and alkaline earth metals. However, the subsequent B-cation substitution, i.e., Ti by Fe in the present series also modifies the charge carrier concentration, charge transfer resistance and carrier lifetime that alters the expected photocatalytic activity solely based on dye adsorption.

It is clear from previous reports that $\mathrm{Bi}_{5} \mathrm{SrTi}_{4} \mathrm{FeO}_{18}$ exhibits higher activity compared to that of $\mathrm{Bi}_{6} \mathrm{Ti}_{3} \mathrm{Fe}_{2} \mathrm{O}_{18}$ and the extent of dye adsorption is a crucial factor for the enhanced photocatalysis. The introduction of one $\mathrm{Sr}$ in place of lone pair active $\mathrm{Bi}$ in the five-layer perovskite modifies the overall octahedral distortion of the perovskite blocks and enhances the electric polarization at the terminal octahedra ensuing enhanced surface charge and dye adsorption. While an increase in the photocatalytic activity is observed on increasing amount of $\mathrm{Fe}$ in $\mathrm{Bi}_{5} \mathrm{SrTi}_{4} \mathrm{FeO}_{18}$ to $\mathrm{Fe}_{1.75}$, it decreases sharply in $\mathrm{Bi}_{6} \mathrm{Ti}_{3} \mathrm{Fe}_{2} \mathrm{O}_{18}$ (compound without $\mathrm{Sr}$ ). Interestingly, the compound with $\mathrm{Fe}_{1.5}$ shows the highest extent of dye adsorption among all the compounds in the series but does not show the highest activity. Therefore, it brings out the role of other competing factors into play when it comes to the overall rate of photocatalytic dye degradation. The compound with $\mathrm{Fe}_{1.25}$ shows the slowest degradation activity due to its relatively high rate of carrier recombination and shorter carrier lifetime and high $R_{\mathrm{CT}}$ together with smaller extent of dye adsorption. In the case of the $\mathrm{Fe}_{1.5}$ compound, the highest extent of dye adsorption is observed but it shows moderate activity due to its higher rate of carrier recombination (increased PL intensity), high $R_{\mathrm{CT}}$ and lower lifetime of charge carriers as compared to the $\mathrm{Fe}_{1.75}$ compound. Despite showing an intermediate dye adsorption, the $\mathrm{Fe}_{1.75}$ compound shows the highest activity due to the combined effect of reduced carrier recombination, higher carrier lifetime and enhanced charge transport (smallest $R_{\mathrm{CT}}$ ). It is presumed that the dominance of the above factors has originated from the internal electric fields. In addition to ferroelectric domain polarization, the polar surface terminations due to intergrowth structure of two oppositely charged motifs (fluorite like layers and perovskite blocks) would give rise to an in-built electric field in the compounds. Moreover, the local octahedral distortions of terminal octahedra at surfaces and surface adsorption would modify the fields. The built-in electric field is believed to be altered by the compositional disorder of $\mathrm{Sr}$ at the $\left[\mathrm{Bi}_{2} \mathrm{O}_{2}\right]^{2+}$ layer. Structure refinements enabling Sr disorder at the $\left[\mathrm{Bi}_{2} \mathrm{O}_{2}\right]^{2+}$ layer indicate the presence of a higher extent of $\mathrm{Sr}$ disorder with increasing $\mathrm{Sr}$ content in the compounds, i.e., $\mathrm{Fe}_{1.75}<\mathrm{Fe}_{1.5}<\mathrm{Fe}_{1.25}$ (Table S9, ESI $\dagger$ ). An increase in $\mathrm{Sr}$ disorder would decrease the charges on the structural motifs, thereby reducing the internal field. The $\mathrm{Sr}$ disorder in the $\left[\mathrm{Bi}_{2} \mathrm{O}_{2}\right]^{2+}$ layer would also cause an expansion of the in-plane lattice due to larger size of Sr. This is evident in the case of $\mathrm{Bi}_{5.25} \mathrm{Sr}_{0.75} \mathrm{Ti}_{3.75} \mathrm{Fe}_{1.25} \mathrm{O}_{18}$, the sample with highest $\mathrm{Sr}$ content in the series.

Furthermore, enhanced photocatalytic activity of $\mathrm{Fe}_{1.75}$ is observed under a biased voltage. The EIS with visible-light illumination and photocatalytic activity of the compound under the external bias can be correlated with an upward band bending effect (Fig. 12). The initial upward band bending effect is due to the internal electric field present in the non-centrosymmetric Aurivillius perovskite phase, while further band bending results with the application of an external electric field. The external field $(1 \mathrm{~V})$ results in partial reversal of ferroelectric domain polarization in the powder samples. The enhanced internal field thus helps in better charge-carrier separation and reduced recombination as the thermal barrier to $\mathrm{e}^{-}-\mathrm{h}^{+}$recombination increases with the increased band bending. It is observed that the impedance is enhanced on the application of a negative bias (Fig. S15, ESI $\dagger$ ) and the positive bias condition favours the charge separation 


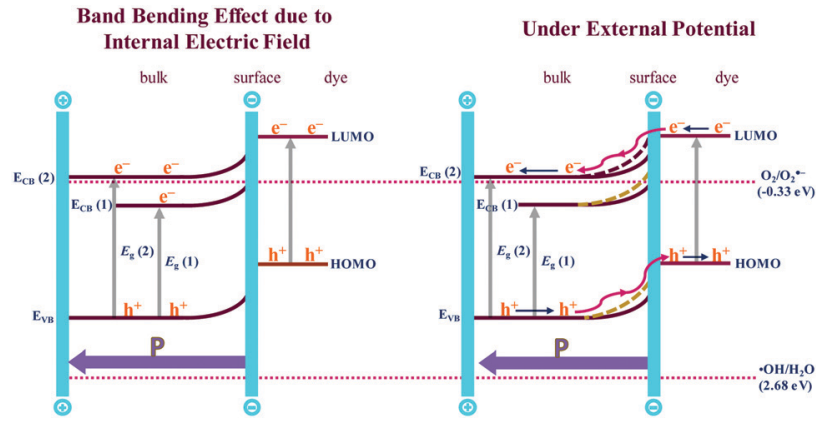

Fig. 12 Schematic diagram showing band bending effect of $n$-type $\mathrm{Bi}_{5.75} \mathrm{Sr}_{0.25} \mathrm{Ti}_{3.25} \mathrm{Fe}_{1.75} \mathrm{O}_{18}$ due to the internal electric field (left) and with the externally applied electric field (right).

and transfer. Despite the samples being powder, the suggested polarization under positive bias is attributed to arise from a preferred orientation of the crystallites in the electrode due to plate-like crystal habits of the layered Aurivillius compounds.

Moreover, the additional band bending (Fig. 12, right) can also enable the participation of the $E_{\mathrm{CB}}(1)$ level for the generation of $\mathrm{O}_{2}^{\bullet-}$, which is forbidden in the absence of an external field. In addition, the bias voltage reduces the probability of trapping and recombination in the semiconductor bulk and increases the hole mobility substantially than that in the absence of an external field. The above factors enhance the photocatalytic activity of $\mathrm{Fe}_{1.75}$ compound substantially under the external bias. The idea can be applied appropriately for engineering the right kind of band bending and enhancement of photocatalytic activity of a semiconductor catalyst with an external electric field by compositional control of cation disorder and octahedral distortion.

\section{Conclusions}

Five-layer Aurivillius perovskites, $\mathrm{Bi}_{6-x} \mathrm{Sr}_{x} \mathrm{Ti}_{3+x} \mathrm{Fe}_{2-x} \mathrm{O}_{18} \quad(x=$ $0.75,0.5,0.25$ and 0.0 ), with different $\mathrm{Ti} / \mathrm{Fe}$ and adjusted $\mathrm{Bi} / \mathrm{Sr}$ ratios were synthesized to study the competing effects of various parameters affecting photocatalytic activity. The compounds crystallized with orthorhombic structure in the non-centrosymmetric F2mm space group. The $\mathrm{Fe}_{1.75}$ compound with smaller $\mathrm{Sr}$ and larger Fe content in the series have exhibited the highest rate of $\mathrm{RhB}$ degradation despite having relatively weak dye adsorption. The octahedral distortion of the SOJT type is found to increase the adsorption capacity of the compounds, being highest in the $\mathrm{Fe}_{1.5}$ compound. Scavenger results and energy level positioning supported $\mathrm{h}^{+}$and $\mathrm{O}_{2}{ }^{\bullet-}$ mediated $\mathrm{RhB}$ degradation. A lower chargetransfer resistance $\left(R_{\mathrm{CT}}\right)$ and enhanced carrier lifetime in $\mathrm{Fe}_{1.75}$ led to an enhanced photocatalytic activity. An external bias of $1 \mathrm{~V}$ led to a further decrease in $R_{\mathrm{CT}}$, and enhanced band bending effect in the compound reduced the thermal barrier to $\mathrm{e}^{-}-\mathrm{h}^{+}$ recombination and enhanced the charge-separation efficiency. The enhanced charge-separation was presumed to originate from partial ferroelectric polarization reversal effects of the powder samples that augment the built-in electric fields. A lower cation disorder at the $\left[\mathrm{Bi}_{2} \mathrm{O}_{2}\right]$ layer of the intergrowth structure in $\mathrm{Bi}_{5.75} \mathrm{Sr}_{0.25} \mathrm{Ti}_{3.25} \mathrm{Fe}_{1.75} \mathrm{O}_{18}$ is believed to have resulted from a higher internal electric field and played an important role in enhancing its activity. The work demonstrates that the cation disorder and octahedral distortion can be controlled via compositional manipulations to achieve higher activities by manipulating internal electric fields. Moreover, the barrier to charge transfer may also be overcome by application of external bias to enhance the photocatalytic activity. Further work involving measurements of polarization and carrier density on single crystals of the compounds would provide quantitative details of internal fields and carrier dynamics for new design strategies of highly efficient photocatalysts.

\section{Conflicts of interest}

There are no conflicts to declare.

\section{Acknowledgements}

SERB, Government of India, through grant number EMR/2016/ 003029, financially supported this work. J. M. thanks the University Grants Commission (UGC), S. K. thanks SERB and the Ministry of Human Resources and Development (MHRD) and P. S. thanks MHRD, Government of India, for providing research fellowships. The authors are thankful to the Department of Metallurgical and Materials Engineering and the Institute Instrumentation Centre (IIC), IIT Roorkee, for providing the facilities.

\section{Notes and references}

1 J. Goldemberg and T. B. Johansson, World energy assessment: overview 2004 update, 2004.

2 M. R. Hoffmann, S. T. Martin, W. Choi and D. W. Bahnemann, Chem. Rev., 1995, 95, 69-96.

3 A. Kudo and Y. Miseki, Chem. Soc. Rev., 2009, 38, 253-278.

4 H. Fujito, H. Kunioku, D. Kato, H. Suzuki, M. Higashi, H. Kageyama and R. Abe, J. Am. Chem. Soc., 2016, 138, 2082-2085.

5 G. Naresh and T. K. Mandal, ACS Appl. Mater. Interfaces, 2014, 6, 21000-21010.

6 C. Wang, X. Zhang, X. Song, W. Wang and H. Yu, ACS Appl. Mater. Interfaces, 2016, 8, 5320-5326.

7 Y. Shiraishi, S. Kanazawa, Y. Kofuji, H. Sakamoto, S. Ichikawa, S. Tanaka and T. Hirai, Angew. Chem., Int. Ed., 2014, 53, 13454-13459.

8 M. Teranishi, R. Hoshino, S. Naya and H. Tada, Angew. Chem., Int. Ed., 2016, 2, 2-7.

9 A. Fujishima and K. Honda, Nature, 1972, 238, 37-38.

10 M. A. Fox and M. T. Dulay, Chem. Rev., 1993, 93, 341-357.

11 O. Legrini, E. Oliveros and A. M. Braun, Chem. Rev., 1993, 93, 671-698.

12 B. Li, C. Lai, G. Zeng, L. Qin, H. Yi, D. Huang, C. Zhou, X. Liu, M. Cheng, P. Xu, C. Zhang, F. Huang and S. Liu, ACS Appl. Mater. Interfaces, 2018, 10, 18824-18836.

13 S. Dong, J. Feng, M. Fan, Y. Pi, L. Hu, X. Han, M. Liu, J. Sun and J. Sun, RSC Adv., 2015, 5, 14610-14630. 
14 J. Yoshimura, Y. Ebina, J. Kondo, K. Domen and A. Tanaka, J. Phys. Chem., 1993, 97, 1970-1973.

15 H. G. Kim, P. H. Borse, J. S. Jang, C. W. Ahn, E. D. Jeong and J. S. Lee, Adv. Mater., 2011, 23, 2088-2092.

16 W. S. Choi, M. F. Chisholm, D. J. Singh, T. Choi, G. E. Jellison and H. N. Lee, Nat. Commun., 2012, 3, 686-689.

17 J. Boltersdorf and P. A. Maggard, ACS Catal., 2013, 3, 2547-2555.

18 W. Bai, C. Chen, J. Yang, Y. Zhang, R. Qi, R. Huang, X. Tang, C.-G. Duan and J. Chu, Sci. Rep., 2015, 5, 17846.

19 G. Naresh and T. K. Mandal, ACS Sustainable Chem. Eng., 2015, 3, 2900-2908.

20 J. W. Tang, Z. G. Zou and J. H. Ye, Catal. Lett., 2004, 92, 53-56.

21 H. G. Kim, D. W. Hwang and J. S. Lee, J. Am. Chem. Soc., 2004, 126, 8912-8913.

22 T. K. Mandal, T. Sivakumar, S. Augustine and J. Gopalakrishnan, Mater. Sci. Eng., B, 2005, 121, 112-119.

23 F. E. Osterloh, Chem. Mater., 2008, 20, 35-54.

24 A. Kudo and Y. Miseki, Chem. Soc. Rev., 2009, 38, 253-278.

25 H. Zhang, G. Chen and D. W. Bahnemann, J. Mater. Chem., 2009, 19, 5089-5121.

26 X. Chen, S. Shen, L. Guo and S. S. Mao, Chem. Rev., 2010, 110, 6503-6570.

27 L. Zhang, Y. Man and Y. Zhu, ACS Catal., 2011, 1, 841-848.

28 B. Dai, Y. Yu, Y. Chen, H. Huang, C. Lu, J. Kou, Y. Zhao and Z. Xu, Adv. Funct. Mater., 2019, 29, 1807934.

29 H. Huang, B. Dai, W. Wang, C. Lu, J. Kou, Y. Ni, L. Wang and Z. Xu, Nano Lett., 2017, 17, 3803-3808.

30 H. Huang, Z. Wang, B. Luo, P. Chen, T. Lin, M. Xiao, S. Wang, B. Dai, W. Wang, J. Kou, C. Lu, Z. Xu and L. Wang, Nano Energy, 2020, 69, 104410.

31 B. Muktha, M. H. Priya, G. Madras and T. N. G. Row, J. Phys. Chem. B, 2005, 109, 11442-11449.

32 Y. Shimodaira, H. Kato, H. Kobayashi and A. Kudo, J. Phys. Chem. B, 2006, 110, 17790-17797.

33 L. Li, P. a Salvador and G. S. Rohrer, Nanoscale, 2014, 6, 24-42.

34 E. C. Subbarao, J. Am. Ceram. Soc., 1962, 45, 166-169.

35 M. S. Haluska and S. T. Misture, J. Solid State Chem., 2004, 177, 1965-1975.

36 B. Frit and J. P. Mercurio, J. Alloys Compd., 1992, 188, 27-35.

37 M. A. Rodriguez, T. J. Boyle, C. D. Buchheit, R. G. Tissot, C. A. Drewien, B. A. Hernandez and M. O. Eatough, Integr. Ferroelectr., 1997, 14, 201-210.

38 S. Sun, Y. Huang, G. Wang, J. Wang, Z. Fu, R. Peng, R. J. Knize and Y. Lu, Nanoscale, 2014, 6, 13494-13500.

39 M. Reddyprakash, S. K. Rout, A. Satapathy, T. P. Sinha and S. M. Sariful, Ceram. Int., 2016, 42, 8798-8803.

40 T. W. Surta, A. Manjón-Sanz, E. K. Qian, R. H. Mansergh, T. T. Tran, L. B. Fullmer and M. R. Dolgos, Chem. Mater., 2017, 29, 7774-7784.

41 H. Kato, M. Hori, R. Konta, Y. Shimodaira and A. Kudo, Chem. Lett., 2004, 33, 1348-1349.
42 H. Fu, C. Pan, W. Yao and Y. Zhu, J. Phys. Chem. B, 2005, 109, 22432-22439.

43 X. Zhao, T. Xu, W. Yao, C. Zhang and Y. Zhu, Appl. Catal., B, 2007, 72, 92-97.

44 Y. Sasaki, H. Nemoto, K. Saito and A. Kudo, J. Phys. Chem. C, 2009, 113, 17536-17542.

45 N. Tian, Y. Zhang, H. Huang, Y. He and Y. Guo, J. Phys. Chem. C, 2014, 118, 15640-15648.

46 B. V. Kumar, M. D. Prasad and M. Vithal, Mater. Lett., 2015, 152, 200-202.

47 A. Etogo, R. Liu, J. Ren, L. Qi, C. Zheng, J. Ning, Y. Zhong and Y. Hu, J. Mater. Chem. A, 2016, 4, 13242-13250.

48 Z. Dai, F. Qin, H. Zhao, J. Ding, Y. Liu and R. Chen, ACS Catal., 2016, 6, 3180-3192.

49 P. Dumrongrojthanath, A. Phuruangrat, S. Thongtem and T. Thongtem, Mater. Lett., 2017, 209, 501-504.

50 C. Yu, Z. Wu, R. Liu, D. D. Dionysiou, K. Yang, C. Wang and H. Liu, Appl. Catal., B, 2017, 209, 1-11.

51 L. Wang, Z. Wang, L. Zhang and C. Hu, Chem. Eng. J., 2018, 352, 664-672.

52 X. Li, Z. Ju, F. Li, Y. Huang, Y. Xie, Z. Fu, R. J. Knize and Y. Lu, J. Mater. Chem. A, 2014, 2, 13366.

53 R. Hailili, Z. Wang, M. Xu, Y. Wang, X. Gong, T. Xu and C. Wang, J. Mater. Chem. A, 2017, 5, 21275-21290.

54 L. Mi, Y. Feng, L. Cao, M. Xue, C. Qin, Y. Huang, L. Qin and H. J. Seo, J. Nanopart. Res., 2018, 20, 2.

55 N. V. Prasad and G. S. Kumar, Mater. Sci. Eng., B, 2004, 108, 194-199.

56 N. V. Prasad and G. S. Kumar, J. Magn. Magn. Mater., 2000, 213, 349-356.

57 W. Łasocha and K. Lewinski, J. Appl. Crystallogr., 1994, 27, 437-438.

58 J. Rodríguez-Carvajal, Phys. B, 1993, 192, 55-69.

59 J.-B. Li, Y. P. Huang, G. H. Rao, G. Y. Liu, J. Luo, J. R. Chen and J. K. Liang, Appl. Phys. Lett., 2010, 96, 222903.

60 S. Sun, W. Wang, H. Xu, L. Zhou, M. Shang and L. Zhang, J. Phys. Chem. C, 2008, 112, 17835-17843.

61 K. Fujihara, S. Izumi, T. Ohno and M. Matsumura, J. Photochem. Photobiol., A, 2000, 132, 99-104.

62 L. Jiang, X. Yuan, G. Zeng, J. Liang, Z. Wu, H. Yu, D. Mo, H. Wang, Z. Xiao and C. Zhou, J. Colloid Interface Sci., 2019, 536, 17-29.

63 P. M. Wood, FEBS Lett., 1974, 44, 22-24.

64 S. Rani, G. Naresh and T. K. Mandal, Dalton Trans., 2020, 49, 1433-1445.

65 M. E. Fleet, Mineral. Mag., 1976, 40, 531-533.

66 M. Kunz and I. David Brown, J. Solid State Chem., 1995, 115, 395-406.

67 Ismunandar, T. Kamiyama, A. Hoshikawa, Q. Zhou, B. J. Kennedy, Y. Kubota and K. Kato, J. Solid State Chem., 2004, 177, 4188-4196.

68 G. Naresh, J. Malik, V. Meena and T. K. Mandal, ACS Omega, 2018, 3, 11104-11116. 